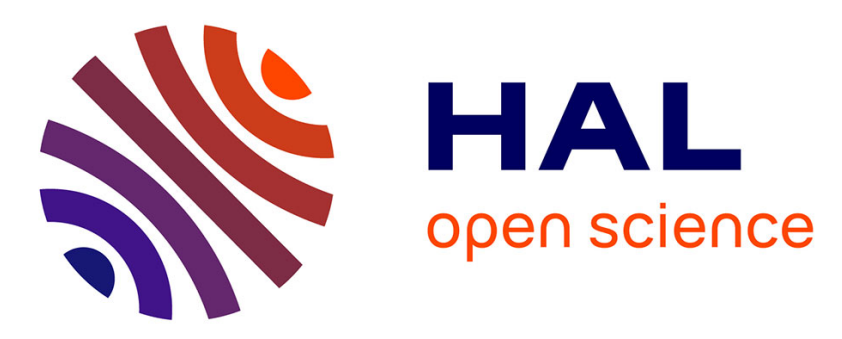

\title{
A Bayesian ensemble of sensitivity measures for severe accident modeling
}

\author{
Hoseyni Seyed Mohsen, Francesco Di Maio, Matteo Vagnoli, Enrico Zio, \\ Mohammad Pourgol-Mohammad
}

\section{- To cite this version:}

Hoseyni Seyed Mohsen, Francesco Di Maio, Matteo Vagnoli, Enrico Zio, Mohammad PourgolMohammad. A Bayesian ensemble of sensitivity measures for severe accident modeling. Nuclear Engineering and Design, 2015, 295, pp.182-191. 10.1016/j.nucengdes.2015.09.021 • hal-01265901

\section{HAL Id: hal-01265901 \\ https://hal.science/hal-01265901}

Submitted on 1 Feb 2016

HAL is a multi-disciplinary open access archive for the deposit and dissemination of scientific research documents, whether they are published or not. The documents may come from teaching and research institutions in France or abroad, or from public or private research centers.
L'archive ouverte pluridisciplinaire HAL, est destinée au dépôt et à la diffusion de documents scientifiques de niveau recherche, publiés ou non, émanant des établissements d'enseignement et de recherche français ou étrangers, des laboratoires publics ou privés. 


\title{
A BAYESIAN ENSEMBLE OF SENSITIVITY MEASURES FOR SEVERE ACCIDENT MODELING
}

\author{
Seyed Mohsen Hoseyni ${ }^{1}$, Francesco Di Maio*2, Matteo Vagnoli ${ }^{2}$, Enrico Zio ${ }^{2,3}$, Mohammad \\ Pourgol-Mohammad ${ }^{4}$ \\ ${ }^{1}$ Department of Basic Sciences, East Tehran Branch, Islamic Azad University \\ Tehran, Iran \\ ${ }^{2}$ Energy Department, Politecnico di Milano \\ Via La Masa 34, 20156 Milano, Italy \\ francesco.dimaio@polimi.it \\ ${ }^{3}$ Chair on System Science and Energetic Challenge \\ Fondation EDF - Electricite de France \\ Ecole Centrale, Paris, and Supelec, Paris, France \\ 4 Department of Mechanical Engineering, Sahand University of Technology \\ Tabriz, Iran
}

\begin{abstract}
In this work, a sensitivity analysis framework is presented to identify the relevant input variables of a severe accident code, based on an incremental Bayesian ensemble updating method. The proposed methodology entails: i) the propagation of the uncertainty in the input variables through the severe accident code; ii) the collection of bootstrap replicates of the input and output of limited number of simulations for building a set of Finite Mixture Models (FMMs) for approximating the probability density function (pdf) of the severe accident code output of the replicates; iii) for each FMM, the calculation of an ensemble of sensitivity measures (i.e., input saliency, Hellinger distance and Kullback-Leibler divergence) and the updating when a new piece of evidence arrives, by a Bayesian scheme, based on the Bradley-Terry model for ranking the most relevant input model variables. An application is given with respect to a limited number of simulations of a MELCOR severe accident model describing the fission products release in the LP-FP-2 experiment of the Loss Of Fluid Test (LOFT) facility, which is a scaled-down facility of a pressurized water reactor (PWR).
\end{abstract}

Keywords: Nuclear Safety, Severe accident modelling, Sensitivity Analysis, Finite Mixture Models, Bayesian Ensemble, Bootstrap Method. 


\section{INTRODUCTION}

Simulation codes used for thermal hydraulic $(\mathrm{TH})$ or severe accident calculations are very complex and, therefore, computationally burdensome. For this reason, one attempts to perform sensitivity analysis with minimal computational burden, for an effective dimensionality reduction of input variables, which can speed up calculations without diminishing the code capability of representing the real system response. Sensitivity analysis can, indeed, inform on the input variables most affecting the simulation results of the system results [Saltelli, 2002; Pourgol-Mohammad, 2009; Di Maio et al., 2014]. However, most sensitivity analysis methods can turn to be themselves computationally expensive [Secchi et al., 2008; Carlos et al., 2013; Di Maio et al., 2014; Pengfei et al., 2014].

In this work, the focus is on the implementation of a sensitivity analysis method to perform input variables ranking with an affordable computational effort. Different solutions have been proposed in literature. One could make theoretical efforts for developing insightful simple novel sensitivity measures [Liu et al., 2010; Plischke et al., 2013] or resort to traditional sensitivity measures (such as Variance Decomposition method [Helton, 1993; MacKay, 1995; Borgonovo, 2007; Cadini et al., 2007], Sobol Indices [Sobol et al., 1995; Saltelli et al., 1999]) in combination with surrogate models like Artificial Neural Networks (ANNs) [Cadini et al., 2007] and Response Surface Methods (RSMs) [Hoseyni et al., 2014], where the idea is to use the surrogate models as substitutes of the complex simulation codes, in the quantification of the contribution of each parameter to the model output.

Alternatively, a relatively novel methodology for global sensitivity analysis is proposed in [Carlos et al., 2013; Di Maio et al., 2015] that does not resort to any surrogate model. The sensitivity results are drawn directly by analyzing Finite Mixture Models (FMMs) built on the results of an uncertainty propagation of the input variables uncertainties onto the simulation code. Ensemble of sensitivity measures can be used [Di Maio et al., 2014], where a set of alternative input variable rankings are aggregated to overcome possible misjudgments of the single sensitivity measure in case of a limited number of code runs. The aggregation methods commonly used are the "majority voting" and "sum" [Kukkonen et al., 2007], where the former consists in assigning the ranking position to each input variable which has been voted by the majority of the calculated sensitivity measures, whereas the latter aggregates by taking the sum of the ranking positions for each input variable provided by the individual sensitivity measures and, then, sorts them with respect to their scores.

The limitations of these two traditional aggregation strategies are that "majority voting" fails in the case of no agreement among the ranking orders provided by the different sensitivity measures, whereas the "sum" strategy fails when the evidence of the superior capability of one (or more) sensitivity measure(s) cannot be accommodated over the remaining ones, within its rigorous 
assignment of equal weights (equal preferences) to the outcomes of the sensitivity measures considered.

In this work, a novel sequential Bayesian approach is embedded into the framework of ensemblebased sensitivity analysis proposed in [Di Maio et al., 2014] for the aggregation of sensitivity measures, so as to overcome the above-mentioned deficiencies. The proposed sensitivity analysis approach is applied to the LP-FP-2 severe accident following a Loss Of Coolant Accident (LOCA) of the Loss of Fluid Test (LOFT) facility, which is a scaled-down facility of a Pressurized Water Reactor (PWR). The most relevant input variables affecting the output of the MELCOR severe accident model are identified.

The novel sequential approach resorts to the FMMs built based on a limited number of code simulations for approximating the probability density function (pdf) of the severe accident model output variables (i.e., the fission products releases during the LOCA) and uses three alternative sensitivity measures, input saliency [Law et al., 2004], Hellinger distance [Gibbs et al., 2002; Diaconis et al., 1982] and Kullback-Leibler divergence [Gibbs et al., 2002; Diaconis et al., 1982], to identify the input variables bearing the most pronounced effects on the output variable, and make their aggregated ranking.

The novelty of the Bayesian aggregation stems from the capability of providing weights to the sensitivity measures and aggregating them according to the degree of belief that one could have on the calculated measures. In other words, the evidence that one sensitivity measure overcomes the others (i.e., the larger degree of belief/weight on one sensitivity measure over the others) is, in principle, obtained by performing a set of sequential experiments with the same Design Of Experiments (DOEs). When the sequential experiments cannot be performed, one could resort to bootstrapped samples of the original outcomes of the experiments to build the FMMs replicates. For each new FMM, the values of sensitivity measures are calculated and treated as the new data (under the same DOE) within a Bayesian updating scheme of the original sensitivity values. A Bradley-Terry model is devised for Bayesian updating [Weng et al., 2011] of the sensitivity measures of the provided input variable ranking orders, that is a full-pair comparison scheme for on-line ranking of multiple players (e.g., input variables) in successive matches (i.e., experiments).

The paper is organized as follows. Section 2 discusses the principles of the ensemble-based sensitivity analysis along with the description of FMMs and the three sensitivity measures used. In Section 3, a definition is given for the Bayesian aggregation approach, the bootstrap technique and the updating rules. Section 4 presents the case of study. Section 5 analyses the results of the implementation of the proposed Bayesian ensemble strategy on the case of study. Conclusions are made in Section 6. 


\section{Ensemble-based sensitivity analysis}

In this Section, a brief summary is provided of the ensemble-based approach for sensitivity analysis presented in [Di Maio et al., 2014]. As shown in Fig. 1, the idea is to rely on a limited number $N$ of code simulations to build a FMM that reproduces the multimodal pdf of the output variable (Section 2.1). In order to identify the input variables with most pronounced effects on the severe accident model output variable, three alternative sensitivity measures are computed (i.e., input saliency [Law et al., 2004], Hellinger distance [Gibbs et al., 2002; Diaconis et al., 1982] and Kullback-Leibler divergence [Gibbs et al., 2002; Diaconis et al., 1982]) (Section 2.2). The reliability of the sensitivity measures is quantified by the sequential Bayesian scheme that aggregates the rankings according to the degree of belief that one could have on the calculated measures (Section 2.3).

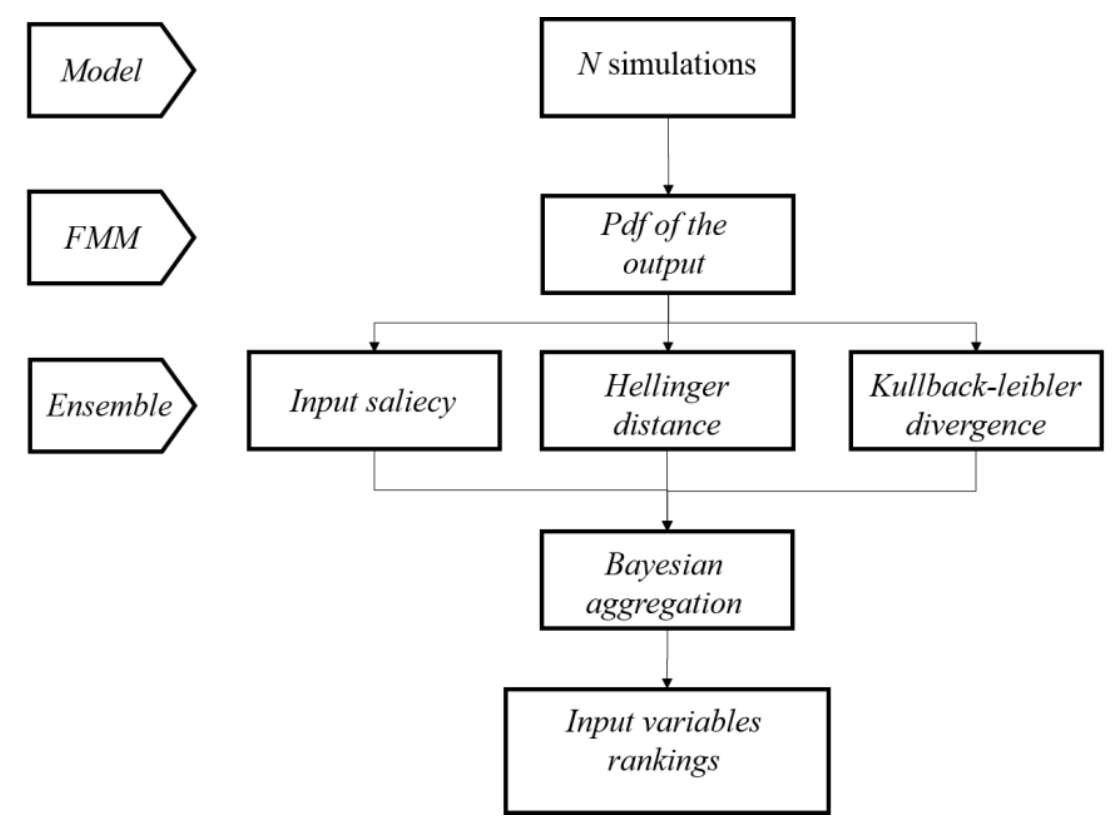

Fig.1 Flowchart of ensemble-based methodology

\subsection{FMM model}

Let $y$ represent the output of a complex code model $m$, viz: 


$$
y=m\left(x_{1} ; x_{2} ; \ldots ; x_{1} ; \ldots ; x_{\mathrm{D}}\right) \quad l=1, \ldots, \mathrm{D}
$$

where $x_{l}$ is the realization of the l-th input variable of the model, whose inputs are distributed with pdf $f\left(x_{l}\right)$. The random output variable $y$ follows a finite mixture density $f(y)$ with $K$ models if [Carlos et al., 2013]:

$$
f(y)=\sum_{k=1}^{K} \pi_{k} f_{k}\left(y \mid \theta_{k}\right) \quad \text { with } \forall k, \pi_{k} \geq 0
$$

where $f_{k}\left(y \mid \theta_{k}\right)$ are $K$ different probability density functions, $\theta_{k}$ is the set of parameters of the $k$-th model of the mixture and $\pi_{k}$ are the mixing probabilities that satisfy:

$$
\sum_{k=1}^{K} \pi_{k}=1
$$

The Expectation Maximization (EM) algorithm [Mc Lachlan et al., 2000] is used to fit $f(y)$ to the $N$ available data $y=\left(y_{1}, \ldots, y_{N}\right), i=1, \ldots, N$ and for the identification of parameters $\theta$ and $\pi$ [Di Maio et al., 2014]. Once the parameters $\theta$ and $\pi$ of the mixture models are known, the best approximation of the pdf of the output of the model is completely characterized with small number of code simulations.

\subsection{Sensitivity Measures}

\subsubsection{Input saliency}

In the case of independence of input variables, the FMM of Eq. (2) can be rewritten as a function of the $D$ input variables of the model: 


$$
f(y)=\sum_{k=1}^{K} \pi_{k} f_{k}\left(y \mid \theta_{k}\right)=\sum_{k=1}^{K} \pi_{k} m\left(\prod_{l=1}^{D} f\left(x_{l} \mid \theta_{k l}\right)\right)
$$

where $m$ is the severe accident model function and $f\left(x_{l} \mid \theta_{k l}\right)$ is the pdf of the $l$-th input $x$ that determines the output $y$ to belong to the $k$-th cluster. The $l$-th input does not affect the output if its distribution is independent from the cluster, i.e., it follows its common density $q\left(x_{l} \mid \theta_{k l}\right)$ among all the clusters [Pudil et al., 1995; Vaithyanathan et al., 1999]. The decomposition of the term $f\left(x_{l} \mid \theta_{k l}\right)$ in Eq. (4) with weights $\rho_{l}$ into a distribution accounting for the contribution of the $l$-th input in the $k$ th cluster $f\left(x_{l} \mid \theta_{k l}\right)$ and with weight " $1-\rho_{l}$ " in the common distribution $q\left(x_{l} \mid \theta_{k l}\right)$, yields to:

$$
f(y)=\sum_{k=1}^{K} \pi_{k} m\left(\prod_{l=1}^{D} \rho_{l} f\left(x_{l} \mid \theta_{k l}\right)+\left(1-\rho_{l}\right) q\left(x_{l} \mid \lambda_{l}\right)\right)
$$

The saliency $\rho_{l}$ is the importance of the $l$-th input in affecting the output $y$. In fact, if $\rho_{l}$ is large, it means that the input variable distribution varies significantly from one cluster to another of the "natural" clusters corresponding to each model $f_{k}\left(y \mid \theta_{k}\right)$ and, thus, the input is important in determining the variability of the output. On the other hand, if $\rho_{l}$ is small, the inputs follow the common distribution in any cluster and, thus, the input is not relevant in shaping the distribution of the output [Di Maio et al., 2014].

The estimation of the input variable importance $\rho_{l}$ is a model parameter identification problem that does not admit any closed form analytical solution [Figueiredo et al., 2002]; thus, it can be tackled by the Expectation Maximization (EM) algorithm as proposed in [McLachlan et al., 2000; Di Maio et al., 2014].

\subsubsection{Hellinger distance}

The Hellinger distance measures the difference between the pdf of the $l$-th input contributing to shape the $k$-th cluster $f\left(x_{l} \mid \theta_{k l}\right)$ and its common distribution $q\left(x_{l} \mid \theta_{k l}\right)$ [Gibbs et al., 2002; Diaconis et al., 1982] by: 


$$
H_{l k}\left(f\left(x_{l} \mid \theta_{k l}\right), q\left(x_{l} \mid \lambda_{l}\right)\right)=\left[\frac{1}{2} \int_{-\infty}^{+\infty}\left(\sqrt{f\left(x_{l} \mid \theta_{k l}\right)}-\sqrt{q\left(x_{l} \mid \lambda_{l}\right)}\right)^{2} d x\right]^{\frac{1}{2}}=\left[1-\int_{-\infty}^{+\infty}\left(\sqrt{f\left(x_{l} \mid \theta_{k l}\right) q\left(x_{l} \mid \lambda_{l}\right)}\right)^{2} d x\right]^{\frac{1}{2}}
$$

satisfying the inequality $0 \leq H_{l k}\left(f\left(x_{l} \mid \theta_{k l}\right), q\left(x_{l} \mid \lambda_{l}\right)\right) \leq 1$.

The importance of the $l$-th input variable in affecting the output $y$ is computed as:

$$
H L_{l}=\sum_{k=1}^{K} H_{l k}\left(f\left(x_{l} \mid \theta_{k l}\right), q\left(x_{l} \mid \lambda_{l}\right)\right)
$$

The $l$-th input variable is important if $H L_{l}$ is large (in relative terms).

\subsubsection{Kullback-Leibler divergence}

The Kullback-Leibler divergence measures the different information carried by the pdf of the $l$-th input in the $k$-th cluster $f\left(x_{l} \mid \theta_{k l}\right)$ and its common distribution $q\left(x_{l} \mid \theta_{k l}\right)$ [Gibbs et al., 2002; Diaconis et al., 1982]:

$$
K_{l k}\left(f\left(x_{l} \mid \theta_{k l}\right), q\left(x_{l} \mid \lambda_{l}\right)\right)=\int_{-\infty}^{+\infty} f\left(x_{l} \mid \theta_{k l}\right) \log \left(\frac{f\left(x_{l} \mid \theta_{k l}\right)}{q\left(x_{l} \mid \lambda_{l}\right)}\right) d x
$$

with values in $[0, \infty]$.

The importance of the $l$-th input variable in affecting the output $y$ is quantified as:

$$
K L_{l}=\sum_{k=1}^{K} K_{l k}\left(f\left(x_{l} \mid \theta_{k l}\right), q\left(x_{l} \mid \lambda_{l}\right)\right)
$$

The $l$-th input variable is important if $K L_{l}$ is large (in relative terms).

\subsection{Aggregation}


The idea of the ensemble is particularly useful for sensitivity analysis when the number $N$ of code simulations is deliberately kept low to control computational cost. As a consequence, due to the limited quantity of data, individual sensitivity methods would mislead the individual rankings because, as shown in Section 2.2, input saliency, Hellinger distance and Kullback-Leibler divergence would quantify different aspects of the input variables importance. On the other hand, the ensemble approach is expected to generate reliable rankings by agreement among the rankings produced by the individual methods without requiring any additional simulation. Thus, the advantage of the ensemble approach is twofold: it can overcome possible misjudgments of the individual methods when alternative sensitivity measures disagree, and, furthermore, it is possible to gain more confidence on the ranking when they agree.

The central issue is to decide how to aggregate the sensitivity ranking outcomes provided by the different methods in the ensemble [Baraldi et al., 2011; Di Maio et al., 2012].

In this paper, we propose a novel Bayesian approach for the aggregation of sensitivity measures results. Classically, in the Bayesian process, for a given prior distribution, one can update the state of knowledge once a new piece of data or evidence becomes available. In the proposed framework, the prior is the value of the sensitivity measures obtained for each parameter from the original FMM; the new evidence (for updating the state of knowledge about the three sensitivity measures) consists in the values of the sensitivity measures derived from the bootstrapping procedure (to mimic the repetitions of experiments under the same DOE).

The ranking results obtained by using the proposed Bayesian approach will be compared with the rankings obtained by traditional ensemble methods such as "majority voting" and "sum" [Kukkonen et al., 2007], where the "majority voting" method fails in the case of no agreement among any of the ranking orders provided by the different sensitivity measures, whereas the "sum" strategy can be proven to be ineffective when the evidence of the superior capability of one (or more) sensitivity measure(s) over the remaining ones cannot be accommodated within its rigorous assignment of equal weights to the outcomes of the involved sensitivity measures.

The benefit of the proposed Bayesian aggregation is to provide weights to the sensitivity measures and aggregate them according to the degree of belief that one could have on the calculated measures. The rationale behind the evidence that one sensitivity measure has larger degree of belief than the others can be obtained (in principle) by performing a set of sequential experiments with the same DOEs; otherwise, when the sequential experiments cannot be performed, one could resort to bootstrapped samples of the original outcomes of the experiments to build the FMMs replicates. In other words, a weight (performance) is assigned to each sensitivity measure in order to i) reward the sensitivity measure with high-ranking stability (reproducibility) during the updating process and ii) 
penalize the measures with fluctuating orders throughout the successive ranking that treats the successively accumulated evidences (i.e., the repeated experiments under the same DOE/bootstrapped repetitions).

\section{The Bayesian aggregation approach}

The proposed approach entails: i) the generation of $B$ bootstrapped samples of the original $N$ code outputs dataset to build $B$ new FMMs replicates (Section 3.1); ii) the evaluation of the three sensitivity measures for each new FMM, that is, thus, treated as a new piece of evidence for the Bayesian updating of the original sensitivity values (Section 3.2) by the Bradley-Terry model [Hunter, 2004; Weng et al., 2011]. Finally, the aggregation of the sensitivity measures is obtained (Section 3.3).

\subsection{The Bootstrap}

The bootstrap is a computer-based method for assessing the accuracy of statistical estimates with very little assumptions or analysis [Efron et al., 1993]. It is used in different frameworks, e.g., in an order statics framework for computing the $\mathrm{TH}$ code uncertainties in order to estimate the safety margins using limited samples of Thermal-Hydraulic (TH) code execution [Pourgol-Mohammad et al., 2007; Zio et al., 2008], or in a framework to propagate uncertainties in TH code calculations [Probst et al., 2006]. The main benefit of bootstrapping approach is to avoid additional computational burden and rely on the available data from the results of uncertainty analysis already performed. In general, 100200 bootstrap replications are enough to obtain a good estimate [Zio et al., 2008].

The basic idea is to generate a number $B$ of bootstrap samples drawn at random with replacement from the original training set of $N$ input/output patterns. The generic bootstrapped sample $b$-th is constituted by the same number $N$ of input/output, although, due to sampling with replacement, some of the input/output will appear more than once whereas some will not appear at all [Efron et al, 1993]. In the proposed approach, the bootstrap technique is employed for generating $B$ times the $N$ replicates from the original dataset of the code as shown in Fig. 1. In other words, each bootstrap process takes samples of the original output dataset and generates a new dataset. The FMMs are built on these new data and used for the calculation of sensitivity measures. Thus, new data/evidences are available for use in the Bayesian updating process.

\subsection{Bayesian updating of the sensitivity measures}


A Bayesian updating process is implemented to derive simple analytic rules for updating the ranking of the code input variables based on the outcomes of the sequential experiments [Weng et al., 2011], based on the Bradley-Terry model (typically used for ranking competitive games) [Hunter, 2004]. The proposed process for updating the sensitivity measures of the input variables (that can be seen as players) uses data provided by the bootstrapped FMMs replicates (that can be seen as consecutive games). With this premises, it can be said that the l-th player (input variable) beats the $q$-th player (input variable) if the first one has lower rank position (higher strength) than the latter. The resulting sensitivity measures represent the games results and, thus, an input variable is more relevant if its sensitivity measure value is higher.

Given a prior distribution of a random variable, Bayesian process allows updating its knowledge when new data or evidence becomes available. In our case, the prior knowledge is the value of the sensitivity measures obtained for each input variable from the original FMM, whereas the new information used for updating the state of knowledge about the three above mentioned sensitivity measures are the values of the sensitivity measures derived from the $B$ replicates of the original $N$ simulations.

Let $\eta_{l}$ be the strength, i.e. the sensitivity measure value of the $l$-th input variable whose value is to be estimated. The Bradley-Terry Bayesian ranking method starts by assuming that $\eta_{l}$ has a prior distribution $N\left(\mu_{l}, \sigma_{l}^{2}\right)$, with $\mu_{l}$ and $\sigma_{l}^{2}$ known [Weng et al., 2011]. Upon a game (bootstrap replication) completion, based on its ranking results $T=\left[T_{1}, \ldots, T_{D}\right]$, the normal distribution $N\left(\mu_{l}, \sigma_{l}^{2}\right)$ is updated to the posterior $N\left(\mu_{l}^{*}, \sigma_{l}^{2^{*}}\right)$, whose updated mean $\mu_{l}^{*}$ and variance $\sigma_{l}^{2^{*}}$ are considered as prior information for the next game up to the $B$ updates.

The procedure for updating $\eta_{1}$ is hereafter described. Let $\bar{Z}=\left[Z_{1}, \ldots, Z_{D}\right]$ be the vector of standard normal variables with probability density function $\Phi_{D}$ [Weng et al., 2011]

$$
Z_{l}=\frac{\eta_{l}-\mu_{l}}{\sigma_{l}}, l=1, \ldots, D
$$

where $D$ is the number of players (input variables). The posterior density distribution of $\bar{Z}$ given the game outcome $\bar{T}=\left[T_{1}, \ldots, T_{D}\right]$, i.e., the ranking results, is [Weng et al., 2011]: 


$$
P(\bar{Z} \mid \bar{T})=\frac{\Phi_{D}(\bar{Z}) f(\bar{Z})}{\int \Phi_{D}(\bar{Z}) f(\bar{Z}) d z}
$$

where $f(\bar{Z})$ is the probability of the game outcome $P(\bar{Z} \mid \bar{T})$ :

$$
f(\bar{Z})=\prod_{l=1}^{D} f\left(Z_{l}\right)
$$

The posterior mean $\mu_{l}^{*}$ and variance $\sigma_{l}^{2^{*}}$ of $\eta_{l}$ for each $l$-th input variable, where $l=1,2, \ldots, D$, are related to $Z_{l}$ [Weng et al., 2011]:

$$
\mu_{l}^{*}=E\left[\eta_{l}\right]=\mu_{l}+\sigma_{l} E\left[Z_{l}\right]=\mu_{l}+\sigma_{l} E\left[\frac{\partial f(\bar{Z}) / \partial Z_{l}}{f(\bar{Z})}\right]
$$

and

$$
\begin{aligned}
\sigma_{l}^{2^{*}}=\operatorname{Var}\left[\eta_{l}\right]=\sigma_{l}^{2} \operatorname{Var}\left[Z_{l}\right] & =\sigma_{l}^{2}\left(E\left[Z_{l}^{2}\right]-E\left[Z_{l}\right]^{2}\right) \\
& =\sigma_{l}^{2}\left(1+\left.E\left[\frac{\partial^{2} f(\bar{Z}) / \partial Z_{l}^{2}}{f(\bar{Z})}\right]\right|_{\bar{Z}=0}-E\left[\frac{\partial f(\bar{Z}) / \partial Z_{l}}{f(\bar{Z})}\right]^{2}\right)
\end{aligned}
$$

The expectation terms in Eqs. (13) and (14) are the average relative changes of $f$ (the probability of the game outcome, that is, the ranking results) with respect to the strength $\eta_{l}$. For example, suppose that input variable 1 beats input variables 2: $f$ is expected to increase for $\eta_{1}$ and the adjustment $\Omega_{1}$ of $\eta_{1}$ is equal to the relative (i.e., normalized on $f(\bar{Z})$ ) rate of change of all input variables strengths with respect to $\eta_{1}$ (i.e, $\partial f(\bar{z}) / \partial Z_{1}$ ), whereas, $f$ is expected to decrease for $\eta_{2}$ and the adjustment $\Omega_{2}$ is negative.

The approximating expectations are, thus, given by:

$$
\mu_{l}^{*} \leftarrow \mu_{l}+\Omega_{l}
$$




$$
\sigma_{l}^{2^{*}} \leftarrow \sigma_{l}^{2} \max \left(1-\Delta_{l}, \kappa\right)
$$

where [Weng et al., 2011]:

$$
\begin{gathered}
\Omega_{l}=\left.\sigma_{l} \frac{\partial f(\bar{Z}) / \partial Z_{l}}{f(\bar{Z})}\right|_{\bar{Z}=0} \\
\Delta_{l}=-\frac{\partial}{\partial z_{l}}\left(\left.\frac{\partial f(\bar{Z}) / \partial Z_{l}}{f(\bar{Z})}\right|_{\bar{Z}=0}\right. \\
\frac{\partial f(\bar{Z}) / \partial Z_{l}}{f(\bar{Z})}=\frac{\partial \log \prod_{l=1}^{D} f\left(Z_{l}\right)}{\partial Z_{l}}
\end{gathered}
$$

and $\kappa$ is a lower bound to ensure a positive $\sigma_{l}^{2}$.

Notice that, the posterior density of $\eta$ is expected to be centered in $\mu$ and, thus, the derivative terms are evaluated at $\bar{Z}=0$ so that the $\eta_{l}$ is replaced by $\mu_{l}$. Furthermore, the right terms of Eqs. (15) and (16) are functions of $\mu_{l}$ and $\sigma_{l}^{2}$, so the updated mean $\mu_{l}^{*}$ and variance $\sigma_{l}^{2^{*}}$ are computed using the current values of $\mu_{l}$ and $\sigma_{l}^{2}$ [Weng et al., 2011].

Finally, we can calculate the probability (after any of the $B$ updates) that variable $l$ is more relevant than variable $q$ as:

$$
P\left(\text { player “l” beats “ } q \text { ”) } \equiv f_{l q}(Z)=\frac{e^{\eta_{l}^{*} / c_{l q}}}{e^{\eta_{l}^{*} / c_{l q}}+e^{\eta_{q}^{*} / c_{l q}}}\right.
$$

where

$$
c_{l q}=\sigma_{l}^{2^{*}}+\sigma_{q}^{2^{*}}+2 \beta
$$

and $\beta$ is a positive constant [Weng et al., 2011].

The pseudo-code of the Bradley-Terry model for full-pair comparison tailored to the ranking updating problem is as follows: 
1. Calculate $\mu_{l}$ and $\sigma_{l}$ with $l=1,2, \ldots, D$.

2. For $l=1,2, \ldots, D$

2.1 For $q=1,2, \ldots, D$, with $q \neq l$,

3. Calculate $\Omega_{l}$ and $\Delta_{l}$ (in Eqs. (17) and (18)) by the following steps:

3.1 Calculate

$$
\begin{gathered}
c_{l q}=\sigma_{l}^{2}+\sigma_{q}^{2}+2 \beta \\
p_{l q}=\frac{e^{\mu_{l} / c_{l q}}}{e^{\mu_{l} / c_{l q}}+e^{\mu_{q} / c_{l q}}} \\
p_{q l}=\frac{e^{\mu_{q} / c_{l q}}}{e^{\mu_{l} / c_{l q}}+e^{\mu_{q} / c_{l q}}} \\
\delta_{q l}=\frac{\sigma_{l}^{2}}{c_{l q}}\left(s-p_{l q}\right) \\
\varepsilon_{q}=\gamma_{q}\left(\frac{\sigma_{l}^{2}}{c_{l q}}\right)^{2} p_{l q} p_{q l}
\end{gathered}
$$

where $s$ is equal to:

$$
s=\left\{\begin{array}{l}
1 \quad \text { if } \operatorname{rank}(q)>\operatorname{rank}(l) \\
1 / 2 \quad \text { if } \operatorname{rank}(q)=\operatorname{rank}(l) \\
0 \quad \text { if } \operatorname{rank}(q)<\operatorname{rank}(l)
\end{array}\right.
$$

and $\gamma_{q}$ is an arbitrary parameter [Weng et al., 2011].

3.2. Calculate

$$
\begin{gathered}
\Omega_{l}=\sum_{q \neq l} \delta_{q} \\
\Delta_{l}=\sum_{q \neq l} \boldsymbol{E}_{q}
\end{gathered}
$$

3.3. The mean value $\mu_{l}$ and the standard deviation $\sigma_{l}$ of the $l$ player are updated using Eqs. (15) and (16), respectively. 
The bootstrapped FMMs replicates and, thus, the input variables sensitivity measures can fluctuate due to the randomness of the sampling (e.g., the uncertainties of the measures in the real experiments with the same DOE) that might lead to wrong ranking results. To counterbalance possible misleading results, we build an ensemble of sensitivity measures introducing a weight (performance) to each sensitivity measure in order to i) reward the sensitivity measure with high-ranking stability (reproducibility) during the updating process and ii) penalize the measures with fluctuating orders throughout the successive ranking that treats the successive accumulated evidences (i.e., the repeated experiments under the same DOE/bootstrapped repetitions). The weight (performance index) of the sensitivity measure, $s m=1,2,3$ for input saliency, Hellinger divergence and Kullback Leibler, respectively, is defined using the Coefficient Of Variation (COV) as:

$$
w_{s m}=\frac{1 / C O V_{s m}}{\sum_{s m=1,2,3}\left(1 / C O V_{s m}\right)}
$$

The COV of each ' $\mathrm{s} m$ ' is the mean value of the COV of the sensitivity measures evaluated on all the code input variables at the last update, accounting for the input variables sensitivity measures variability in relation to their mean value, i.e., COV aims to describe the goodness of each sensitivity measure in terms of the relative sizes of the squared residuals and mean values. The higher the COV, the greater the dispersion in the sensitivity measures evaluated on all the code input variables; whereas, the lower the $\mathrm{COV}$, the smaller the residuals relative to the sensitivity measures mean value. COV is defined as [Zio, 2007]:

$$
C O V_{l}=\frac{\sigma_{l}}{\mu_{l}}
$$

where $l=1,2, \ldots . D$.

Once the weights are calculated for each SA measure, the Bayesian aggregation of their values quantifies for each $l$-th input variable an ensemble of the three SA measures as the weighted sum of the three $\eta_{l}$ :

$$
E S A_{l}=\sum_{s m=1}^{3} w_{s m} \eta_{s m}
$$

Fig. 2 schematically represents the proposed scheme. 


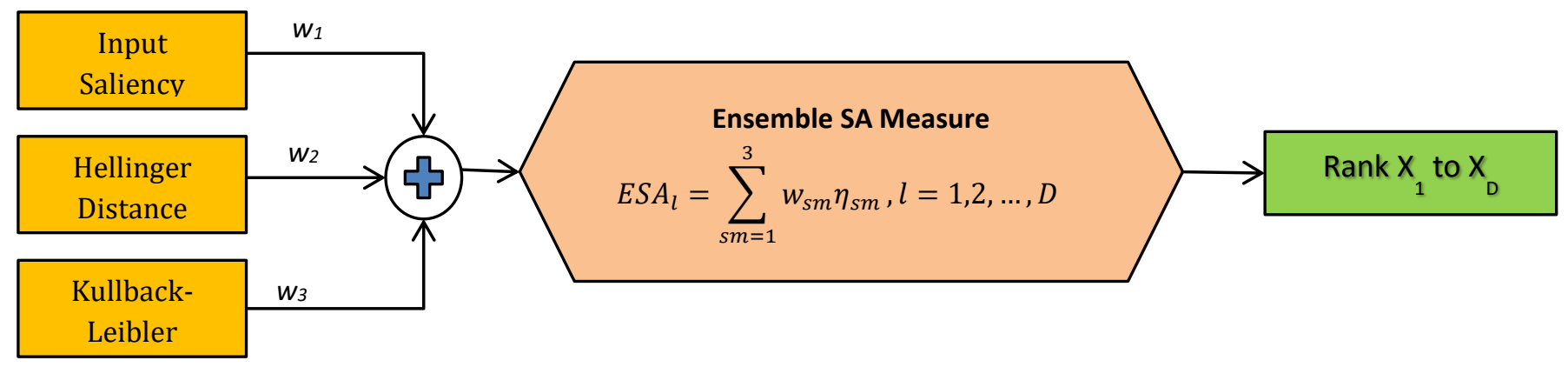

Fig. 2: Bayesian aggregation of SA measures

\section{The LOFT LP-FP-2 experiment}

\subsection{Description of LOFT LP-FP-2 experiment}

The LOFT experimental facility [Kmetyk, 1992] was designed to simulate the major components and system responses of a current-generation Pressurized Water Reactor (PWR) during a LOCA. The experimental subsystems include the reactor vessel, the intact loop, the broken loop, the Blow-down Suppression Tank (BST) system, and the Emergency Core Cooling System (ECCS). The layout of the major LOFT components for test LP-FP-2 is shown in Fig. 3. LOFT is a PWR model with a rated power of $50 \mathrm{MW}_{\text {th }}$, including all systems and components needed to simulate a severely damaged core assembly and to determine the fission product release and transport to the piping system [Lewis et al., 2008]. The LOFT-FP project, completed in 1985 [IRSN-200783, 2007], was conducted by the Idaho National Laboratory (INL/INEL) on an assembly of $121 \mathrm{UO}_{2}$ rods with nuclear heating (inpile) core. It consisted of tests on rod degradation and fission product release, and involved temperatures up to $2400 \mathrm{~K}$. 


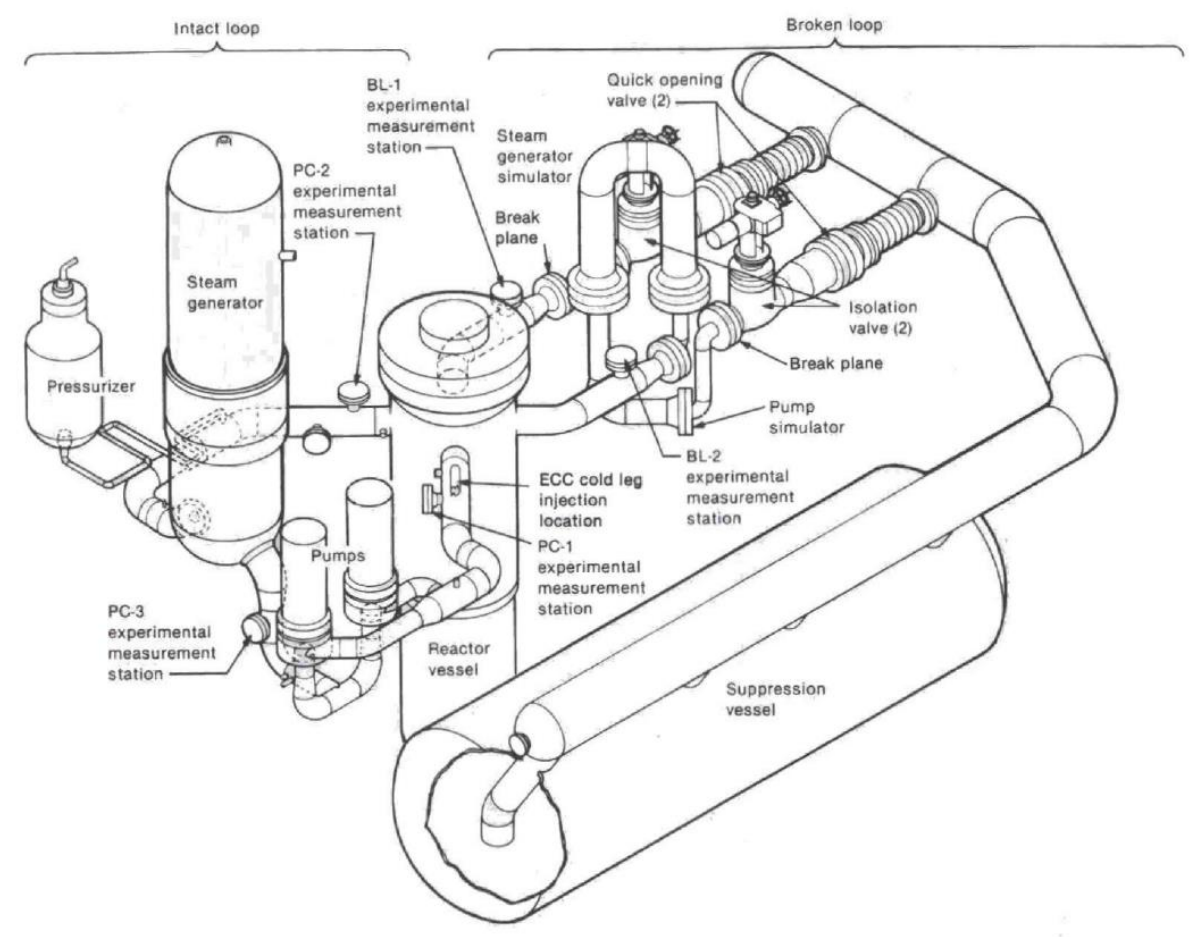

Fig. 3: Schematic Diagram of the LOFT Test Facility for Experiment LP-FP-2 [Kmetyk, 1992]

The specific objectives of the test are to [OECD/NEA, 2001]: i) simulate a medium break LOCA, with delayed operation of the ECCS; ii) attain substantial fuel damage and maintain maximum fuel temperatures above $2100 \mathrm{~K}$ for several minutes; iii) study fission product migration along the leak path, which represents the Low-Pressure coolant Injection System (LPIS) pipe connected to the hot leg.

This test has provided very interesting data on core degradation and fission product release and transport in a V-type LOCA sequence, under low system pressure, in a decay-heated bundle, and at a larger scale compared with other bundle in-reactor tests. The test confirmed the damage zones observed in Three Mile Island-2, showed the inability of blockages to stop fuel oxidation, and showed significant damage and enhanced hydrogen production occurrence during the reflood. Quantitative measurements of fission product deposition have been made. The test has shown a dominance of silver iodide (AgI) as chemical form of Iodine (I).

To show the applicability of the proposed framework of Section 3 for the sensitivity analysis of the MELCOR code simulating the prediction of the fission product release in LOFT LP-FP-2 experiment, the "Xenon release fraction" under CORSOR modeling option is considered as output $y$ of interest. MELCOR model nodalization is shown in Fig. 4. A total of $N=100$ runs of the code have been done, each one with a different batch of input variables values sampled from the distributions listed in Table 1 [Hoseyni et al., 2014]. The very small number of samples has been chosen with the aim of challenging the proposed method: as we shall see in what follows, we will be able, by bootstrapping 
$B=100$ times the $N$ samples, to collect different replicates of the code output under the same DOE and to provide an effective input variables ranking as compared with other aggregation methods of literature that have been proposed for sensitivity analsysis [Di Maio et al., 2014].

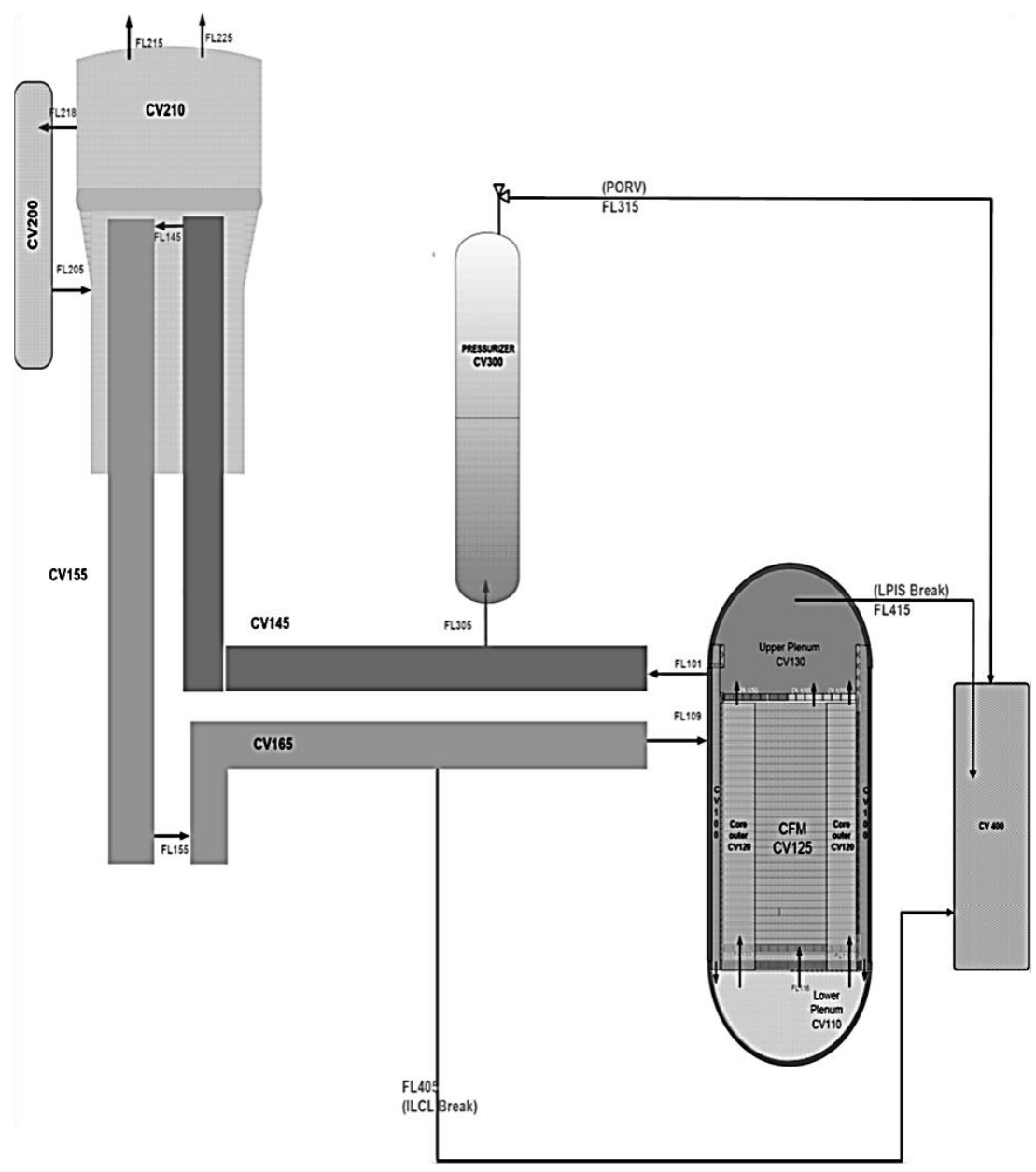

Fig. 4 MELCOR model nodalization for LOFT-LP-FP2 experiment

Table 1: Uncertain Input parameters of LOFT MELCOR model and their distributions [Hoseyni et al., 2014]

\begin{tabular}{|c|l|l|c|c|c|}
\hline & \multicolumn{1}{|c|}{$\begin{array}{c}\text { Input } \\
\text { variables }\end{array}$} & \multicolumn{1}{|c|}{ Description } & $\begin{array}{c}\text { Type of } \\
\text { distribution }\end{array}$ & Mean value $\boldsymbol{\mu}$ & $\begin{array}{c}\text { Standard } \\
\text { deviation } \boldsymbol{\sigma}\end{array}$ \\
\hline $\mathbf{1 .}$ & POWER & Fission Power (MW) & normal & $28.8 \mathrm{E}+6$ & $0.467 \mathrm{E}+6$ \\
\hline $\mathbf{2 .}$ & SAREA-1 & ILCL break flow area (FL415) & normal & 0.000683 & $3.48469 \mathrm{E}-05$ \\
\hline $\mathbf{3 .}$ & SAREA-2 & LPIS break flow area (FL405) & normal & 0.000683 & $3.484 \mathrm{E}-05$ \\
\hline $\mathbf{4 .}$ & BDC-1 & ILCL Break Discharge Coefficient (FL415) & normal & 1 & 0.067 \\
\hline $\mathbf{5 .}$ & BDC-2 & LPIS Break Discharge Coefficient (FL405) & normal & 1 & 0.067 \\
\hline $\mathbf{6 .}$ & BLC-1 & ILCL Break Loss Coefficient (FL415) & normal & 1.005 & 0.507 \\
\hline $\mathbf{7 .}$ & BLC-2 & LPIS Break Loss Coefficient (FL405) & normal & 1.005 & 0.507 \\
\hline $\mathbf{8 .}$ & RVF1 & Core radiation view factors & normal & 0.03 & 0.005 \\
\hline $\mathbf{9}$ & RVF2 & Core radiation view factor 2 & normal & 0.22 & 0.01 \\
\hline
\end{tabular}




\begin{tabular}{|c|c|c|c|c|c|c|}
\hline 10. & ZMLT & \multicolumn{2}{|c|}{ Zircaloy Melt Temperature } & normal & 2200 & 51.02 \\
\hline 11. & DSF & \multicolumn{2}{|c|}{ Dynamic Shape Factor (RNMS000) } & normal & 1 & 0.408 \\
\hline 12. & ASF & \multicolumn{2}{|c|}{ Agglomeration Shape Factor (RNMS000) } & normal & 1 & 0.408 \\
\hline 13. & SC7101-1 & CORSOR parameter-1 & C7101 $(1,1,1)$ & normal & 900 & 5.102 \\
\hline 14. & SC7101-2 & CORSOR parameter-2 & C7101(1,1,2) & normal & 900 & 5.102 \\
\hline 15. & SC7101-3 & CORSOR parameter-3 & C7101 $(1,1,4)$ & normal & 900 & 5.1026 \\
\hline 16. & SC7101-4 & CORSOR parameter-4 & C7101 $(2,1,1)$ & normal & 1400 & 20.408 \\
\hline 17. & SC7101-5 & CORSOR parameter-5 & C7101(2,1,2) & normal & 1400 & 20.408 \\
\hline 18. & SC7101-6 & CORSOR parameter-6 & C7101 $(2,1,4)$ & normal & 1400 & 20.408 \\
\hline 19. & SC7101-7 & CORSOR parameter-7 & C7101 $(3,1,1)$ & normal & 2200 & 25.510 \\
\hline 20. & SC7101-8 & CORSOR parameter- 8 & C7101 $(3,1,2)$ & normal & 2200 & 25.510 \\
\hline 21. & SC7101-9 & CORSOR parameter-9 & C7101 $(3,1,4)$ & normal & 2200 & 25.510 \\
\hline 22. & SC7101-10 & CORSOR parameter-10 & C7101(1,2,1) & normal & $7.02 \mathrm{E}-09$ & $3.581 \mathrm{E}-10$ \\
\hline 23. & SC7101-11 & CORSOR parameter-11 & C7101 $(1,3,1)$ & normal & 0.0088 & 0.00047 \\
\hline 24. & SC7101-12 & CORSOR parameter-12 & C7101(1,2,2) & normal & $7.525 \mathrm{E}-12$ & $3.852 \mathrm{E}-13$ \\
\hline 25. & SC7101-13 & CORSOR parameter-13 & C7101(1,3,2) & normal & 0.0142 & 0.000724 \\
\hline 26. & SC7101-14 & CORSOR parameter-14 & C7101 $(1,2,4)$ & normal & $7.02 \mathrm{E}-09$ & $3.581 \mathrm{E}-10$ \\
\hline 27. & SC7101-15 & CORSOR parameter-15 & C7101(1,3,4) & normal & 0.00886 & 0.000452 \\
\hline 28. & SC7101-16 & CORSOR parameter-16 & C7101(2,2,1) & normal & 0.000000202 & $1.03061 \mathrm{E}-08$ \\
\hline 29. & SC7101-17 & CORSOR parameter-17 & C7101(2,3,1) & normal & 0.00667 & 0.000340306 \\
\hline 30. & SC7101-18 & CORSOR parameter-18 & C7101(2,2,2) & normal & $1.7019 \mathrm{E}-07$ & $2.65357 \mathrm{E}-08$ \\
\hline 31. & SC7101-19 & CORSOR parameter-19 & C7101(2,3,2) & normal & 0.00667 & 0.000340 \\
\hline 32. & SC7101-20 & CORSOR parameter-20 & C7101(2,2,4) & normal & 1.7019E-07 & $2.653 \mathrm{E}-08$ \\
\hline 33. & SC7101-21 & CORSOR parameter-21 & C7101(2,3,4) & normal & 0.00667 & 0.00034 \\
\hline 34. & SC7101-22 & CORSOR parameter-22 & C7101(3,2,1) & normal & 0.0000174 & $8.877 \mathrm{E}-07$ \\
\hline 35. & SC7101-23 & CORSOR parameter-23 & C7101(3,3,1) & normal & 0.0046 & 0.000234 \\
\hline 36. & SC7101-24 & CORSOR parameter-24 & C7101(3,2,2) & normal & 0.0000174 & $8.877 \mathrm{E}-07$ \\
\hline 37. & SC7101-25 & CORSOR parameter-25 & C7101(3,3,2) & normal & 0.0046 & 0.000234 \\
\hline 38. & SC7101-26 & CORSOR parameter-26 & C7101(3,2,4) & normal & 0.0000174 & $8.87755 \mathrm{E}-07$ \\
\hline 39. & SC7101-27 & CORSOR parameter-27 & $\mathrm{C} 7101(3,3,4)$ & normal & 0.0046 & 0.000234 \\
\hline & $\begin{array}{c}\text { Input } \\
\text { variables }\end{array}$ & Descrit & & $\begin{array}{c}\text { Type of } \\
\text { distribution }\end{array}$ & Lower value & Upper value \\
\hline 40. & OPHEIT-1 & Core Flow Path Openin & ights (FL115) & uniform & 0.01 & 1 \\
\hline 41. & OPHEIT-2 & Core Flow Path Opening & ights (FL116) & uniform & 0.01 & 1 \\
\hline 42. & SC1214 & Turbulent Forced Conve & e Flow in Tubes & uniform & 0.0115 & 0.0345 \\
\hline 43. & SC1221 & $\begin{array}{l}\text { Laminar Free Convectio } \\
\text { Vertical Surfaces }\end{array}$ & tween Parallel & uniform & 0.09 & 0.27 \\
\hline 44. & SC1222 & $\begin{array}{l}\text { Turbulent Free Convect } \\
\text { Vertical Surfaces }\end{array}$ & between Parallel & uniform & 0.0325 & 0.0975 \\
\hline 45. & SC1231 & $\begin{array}{l}\text { Forced Convective Flow } \\
\text { Particle }\end{array}$ & er a Spherical & uniform & 0.3 & 0.9 \\
\hline 46. & SC1232 & $\begin{array}{l}\text { Free Convective Flow o } \\
\text { Particle }\end{array}$ & a Spherical & uniform & 0.3 & 0.9 \\
\hline 47. & SC1001-5 & $\begin{array}{l}\text { Zircaloy Oxidation Rate } \\
\text { Coefficients (T lower) }\end{array}$ & nstant & uniform & 1843 & 1863 \\
\hline 48. & SC1001-6 & $\begin{array}{l}\text { Zircaloy Oxidation Rate } \\
\text { Coefficients (T upper) }\end{array}$ & nstant & uniform & 1864 & 1882 \\
\hline 49. & OXTHICK & $\begin{array}{l}\text { Minimum Oxide Shell T } \\
\text { (COR00008) }\end{array}$ & kness & uniform & $1 \mathrm{E}-6$ & $2 \mathrm{E}-4$ \\
\hline
\end{tabular}




\begin{tabular}{|c|l|l|l|l|l|}
\hline $\mathbf{5 0 .}$ & TBLOCK & Time of blockage formation & uniform & 1400 & 1600 \\
\hline
\end{tabular}

\section{Sensitivity analysis results for the LOFT LP-FP-2 model}

Based on the $N=100$ original code runs [Hoseyni et al., 2015], the histogram of Xenon release is shown in Fig. 5, where several maxima can be seen to be approximated by a multimodal analytical distribution. Therefore, in Fig. 5 it is also shown the analytical reconstruction (solid line) of the prior distribution of the Xenon release function, $f(y)$, that has been obtained by the FMM method, where the number $K$ of clusters is set equal to 3 and the parameters values of the $K$ Gaussian models have been obtained by Expectation Maximization algorithm [Carlos et al., 2013]. The choice of using three Gaussian distributions for the FMM parameters identification is the result of a trial and error procedure. An automatic optimization of the number $K$ of distributions in the FMM is also possible [Figueiredo et al., 2002]. As a last remark, it is worth pointing out that the common distributions $q(x)$ used in this application are, instead, uniform distributions.

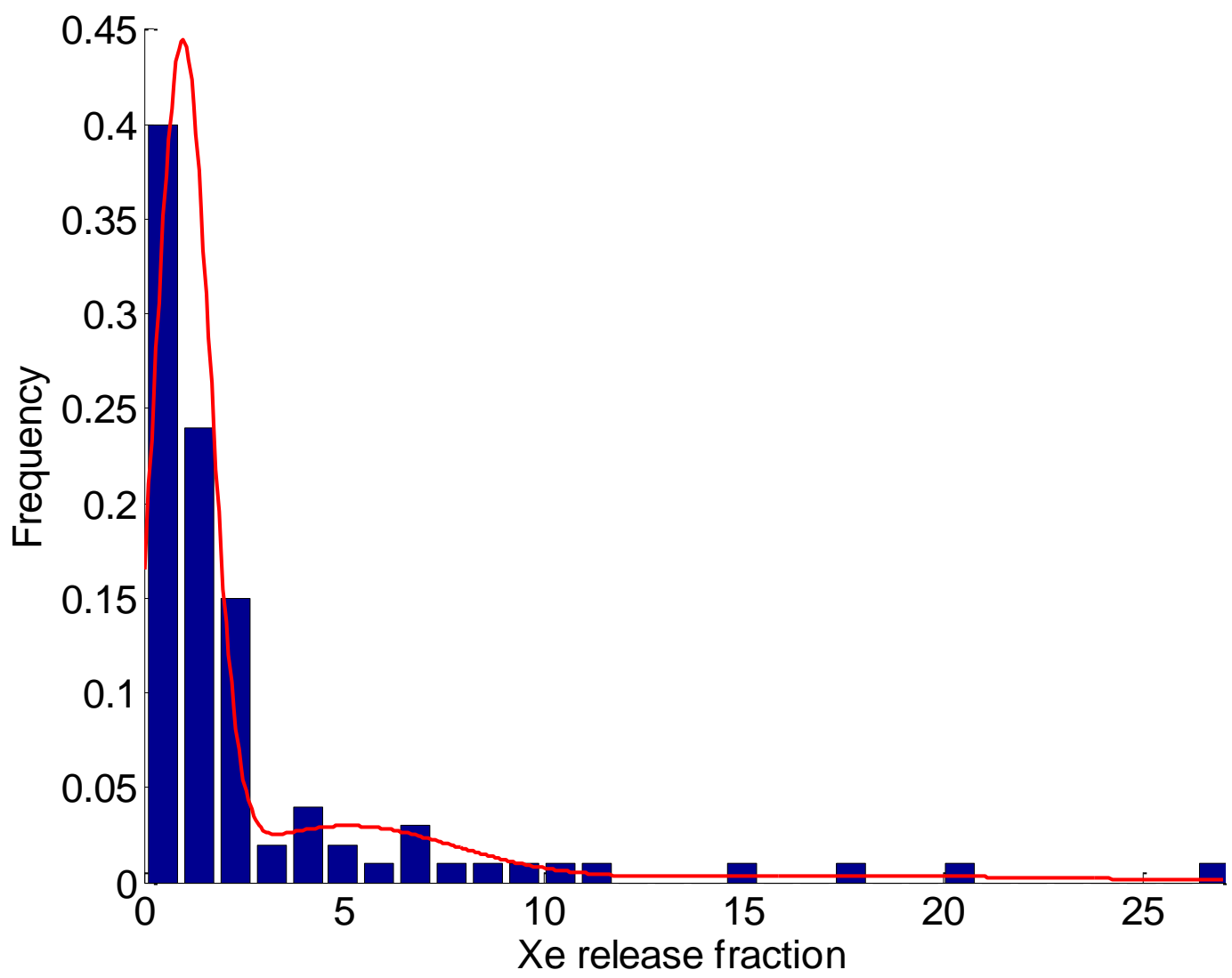

Fig. 5: reconstructed PDF of the model output based on a three-cluster FMM (solid line) for the original code output dataset. 
In order to obtain additional information to provide a realistic evaluation of the Xenon release fraction variability and, thus, an increase of the robustness of the sensitivity analysis results, the original Xenon dataset of code simulations has been replicated by Bootstrap to generate $B$ datasets for a total of $B=100$ bootstrap samples (Section 3.1).

For each $b$-th bootstrap sample, the analytical reconstruction of the $N$ simulations replicates pdf $f(y)$ is performed by FMMs and, thus, the sensitivity measures are evaluated. The new values of sensitivity measures, derived from the $b$-th bootstrap, are used as new evidences for updating the state of knowledge about three sensitivity measures, i.e., the mean value $\mu_{l}$ and standard deviation $\sigma_{l}$ of the sensitivity measures are updated into the posterior mean $\mu_{l}^{*}$ and variance $\sigma_{l}^{2^{*}}$ by the Bradley-Terry algorithm (Section 3.2).

Without loss of generality, the updated mean $\mu_{l}^{*}$ (Eq. 15), updated variance $\sigma_{l}^{2^{*}}$ (Eq. 16) and the updated rank position for input variable $l=19$ as a function of the $b$-th updating step are shown in Fig. 6. Whatever the sensitivity measure considered, Fig. 6 shows that:

1) the variance decreases as the updating procedure proceeds;

2) the mean and, consequently, the rank behave differently from the variance, and fluctuate.

3) the Hellinger distance and Kullback-Leibler divergence show larger stability than input saliency in the updating procedure (i.e., smaller ripples in the plot of the mean). 
Input

Saliency
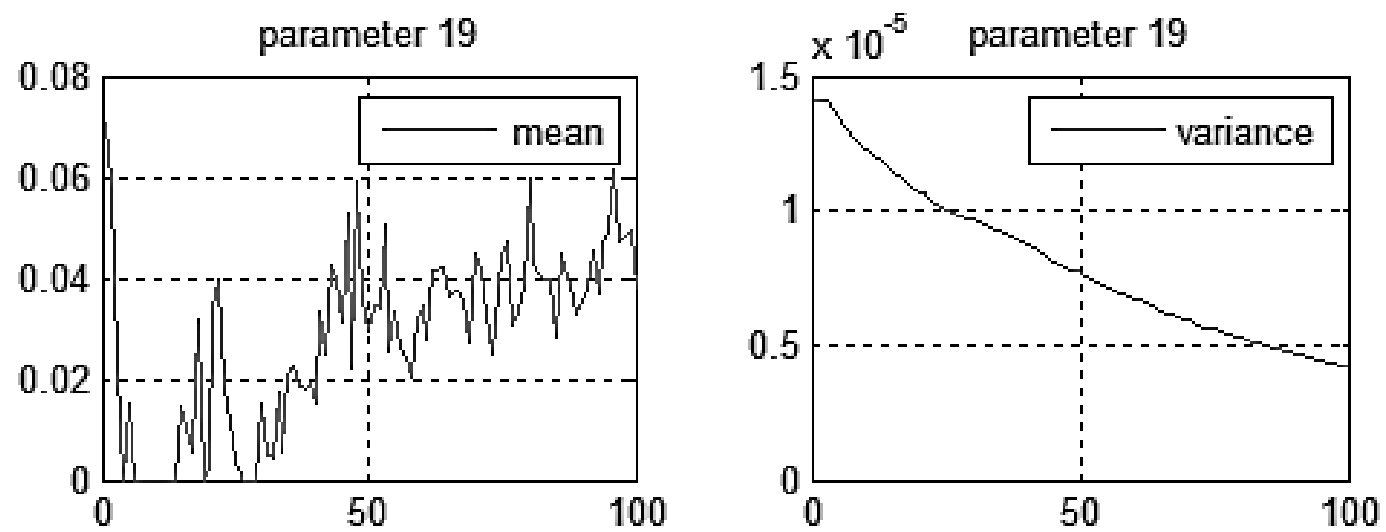

rank of $\mathrm{P} 19$

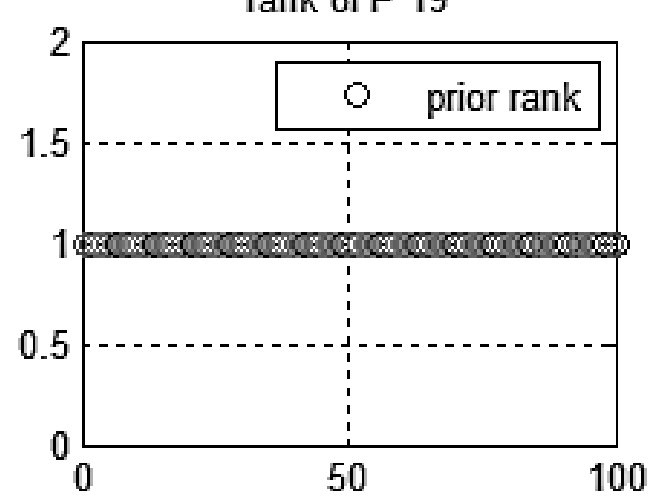

rank of $P 19$

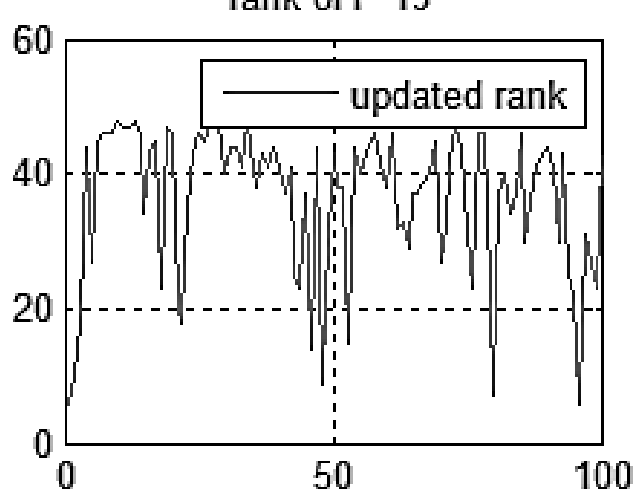

Hellinger
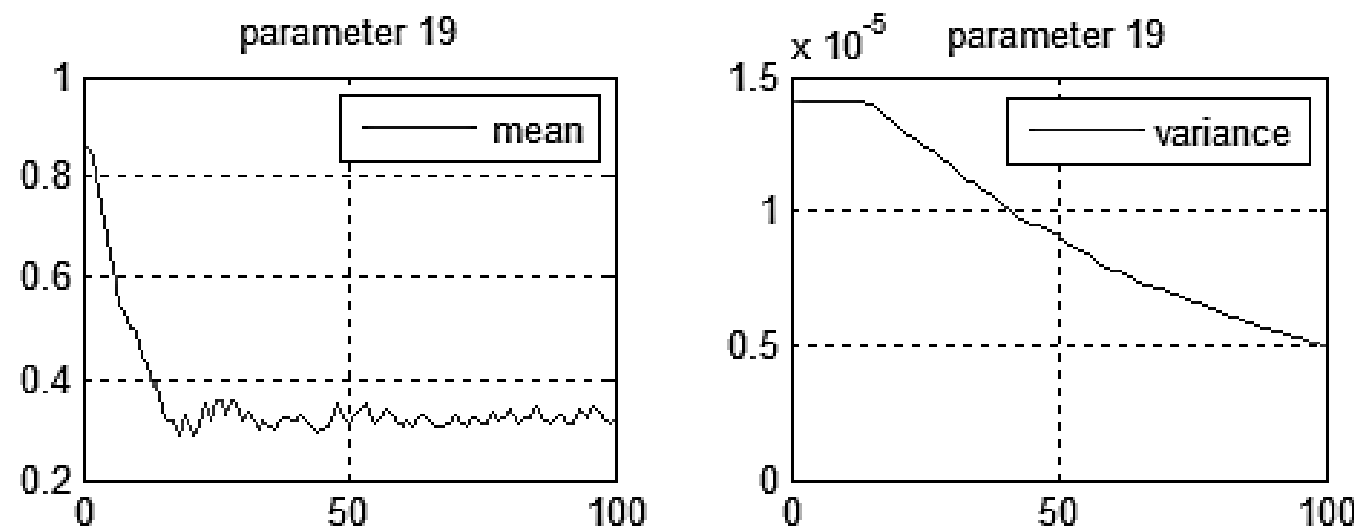

rank of $\mathrm{P} 19$
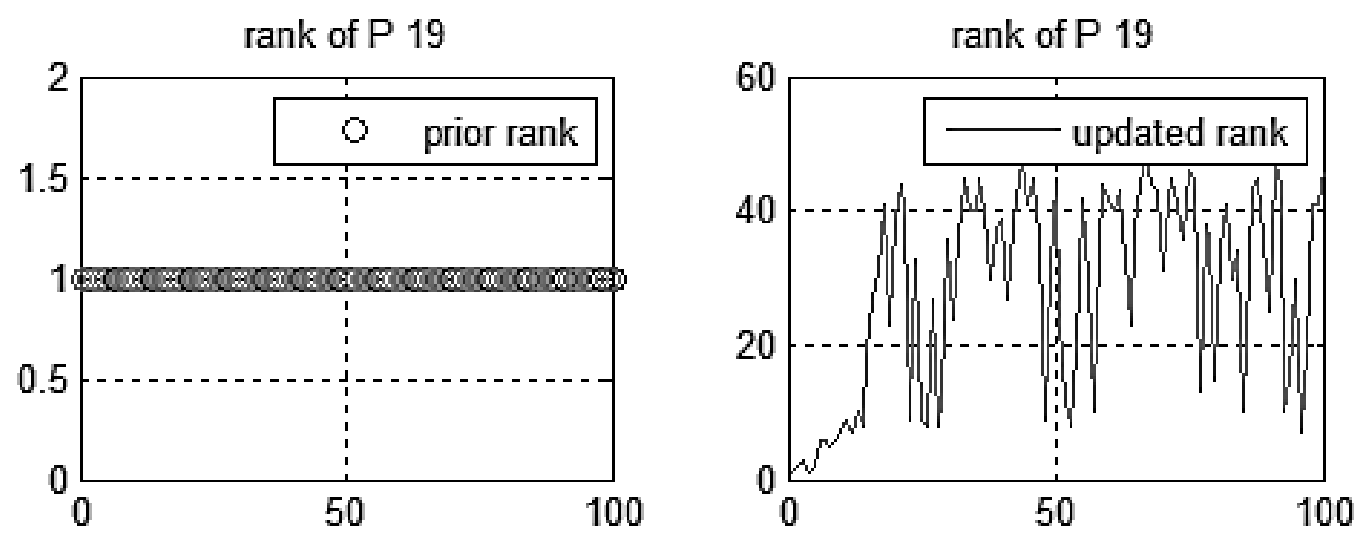
Kulback-

Leibler
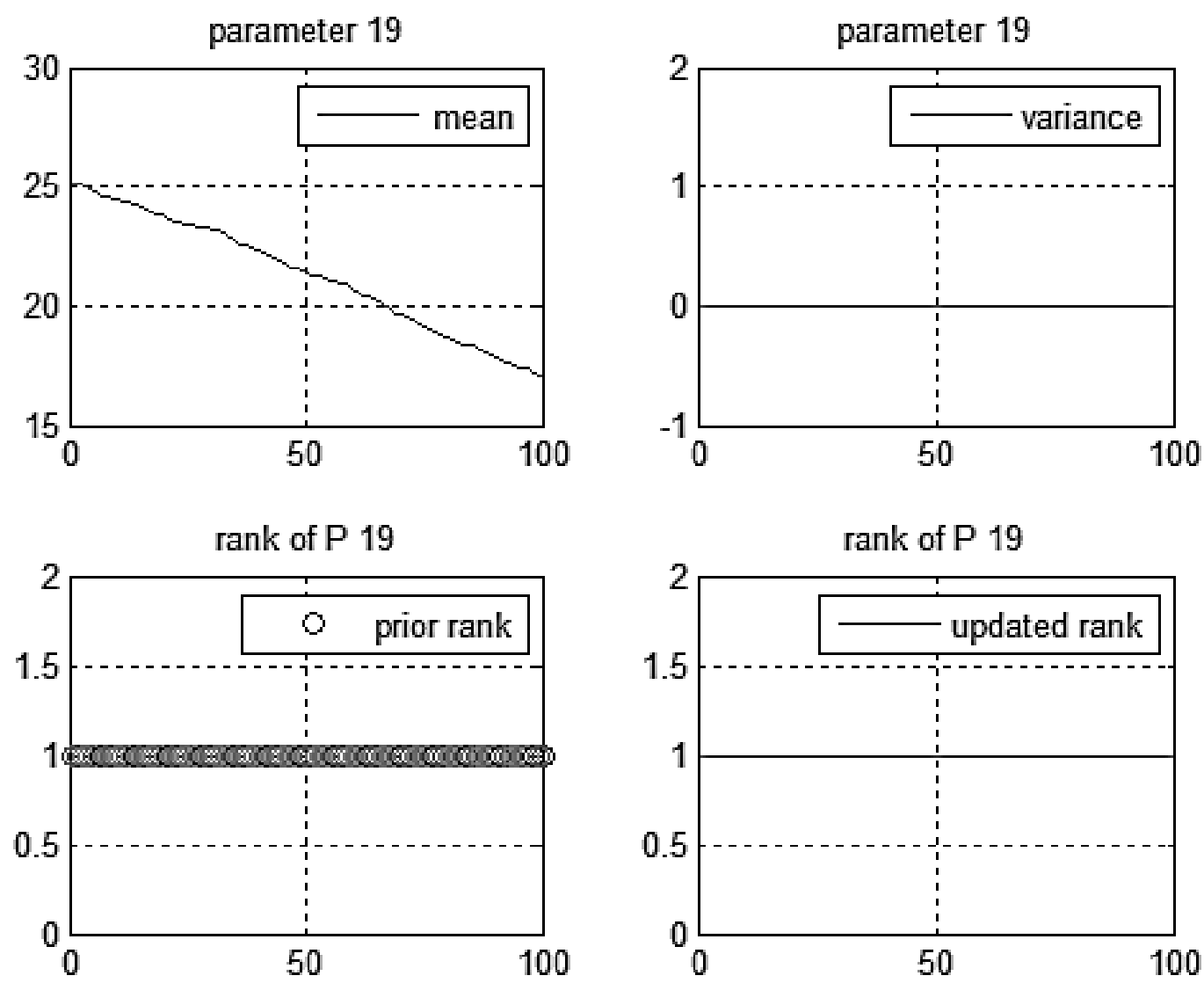

Fig. 6: Updated mean, variance and rank for input variable 19 of Table 1

We, then, perform the Bayesian ensemble by calculating the weighted sum of Eq. (32) with the weights listed in Table 2 (Section 3.3), where the acknowledged better stability of Hellinger distance and Kullback-Leibler divergence with respect to input saliency is rewarded with larger weights, that account, indeed, for the high-ranking stability (reproducibility) of the sensitivity measure during the updating process (as pointed out before in Section 3)).

Table 2: Bayesian weights of SA measures for aggregation

\begin{tabular}{|c|c|}
\hline SA Measure & Assigned weight \\
\hline$\rho$ & 0.0582 \\
\hline HD & 0.4595 \\
\hline KL & 0.4823 \\
\hline
\end{tabular}

The results of the final Bayesian ensemble of sensitivity measures are reported in Table 3 where the first 20 ranking positions are compared by showing the input variables that would have been assigned 
by the traditional sum $\left(\mathrm{R}_{\text {sum }}\right)$ and majority voting $\left(\mathrm{R}_{\mathrm{mv}}\right)$ aggregation approaches [Kukkonen et al., 2007] that are the much easier but approximate methods for sensitivity analysis purposed in [Di Maio et al., 2014].

In fact, it can be seen that, the proposed Bayesian method, $R_{\text {sum }}$ and $R_{m v}$ approaches agree only on the two most important input variables (i.e., the Minimum Oxide Shell Thickness (OXTHICK) and the Agglomeration Shape Factor (ASF)), whereas they disagree on the other input variables. One might argue that $\mathrm{R}_{\text {sum }}$ or $\mathrm{R}_{\mathrm{mv}}$ approaches still remain the best choices due to the fact that among themselves they agree on the first four positions, and to their lower computational burden. In fact, $\mathrm{R}_{\text {sum }}$ and $\mathrm{R}_{\mathrm{mv}}$ are computed using only the $B=1$ original MELCOR output dataset and, thus, any further bootstrap replication is avoided.

However, even if the proposed Bayesian method uses $B=100$ bootstraps of the original MELCOR output dataset (i.e., the computational time is $B$ times larger than for $\mathbf{R}_{\text {sum }}$ and $\mathbf{R}_{\mathrm{mv}}$ ), the Bayesian ensemble aggregation is hereafter shown to be preferable because it holds two fundamental properties of Bayesian processes: i) the memory property and ii) the exchangeability property.

The memory property entails a prior belief (e.g., the value of sensitivity measures obtained for each input variable from the original FMM) to be differently updated depending on the quantity and quality of the available evidence (e.g., the new bootstrap replicates) used for computing the posterior [Friedman et al., 1997]. Consequently, different batches of evidences lead to different posterior distributions (e.g., different sensitivity measures values and ranking positions). Figure 7 shows that the memory property holds in our case: two different batches of evidences of $t=20,40$ successive bootstrap replicates, respectively, are used. Dashed line with circles in Fig. 7 shows the updated aggregated sensitivity measures for input variable 19 for $t=20$ successive updates, whereas dotted line with squares are those for $t=40$ updates: a perfect matching in the updating process of the aggregated sensitivity measures is obtained using the first and the second batch up to the 20-th bootstrap, due to the use of the same cumulated evidence. Contrarily, assuming that the bootstrap replicates are not available up to $t=20$ and that we start calculating from $t=21$ up to $t=40$, the aggregated sensitivity measures at time $\mathrm{t}=40$ differ from those of the batch that exploits all the 40 bootstrap replicates, due to the lack of the first 20 evidences (continuous line with triangles in Fig. 7).

Table 3: Comparison of the input variables ranking position provided by different approaches

\begin{tabular}{|c|c|c|c|}
\hline Rank & Bayesian & $\mathbf{R}_{\text {sum }}$ & $\mathbf{R}_{\mathbf{m v}}$ \\
\hline 1 & OXTHICK & OXTHICK & OXTHICK \\
\hline 2 & ASF & ASF & ASF \\
\hline 3 & SAREA-1 & C7101(3,3,2) & C7101(3,3,2) \\
\hline
\end{tabular}




\begin{tabular}{|c|c|c|c|}
4 & SC1214 & BDC-1 & BDC-1 \\
\hline 5 & C7101(3,3,2) & SAREA-1 & SC1221 \\
\hline 6 & SC1221 & BDC-2 & C7101(1,3,1) \\
\hline 7 & DSF & SC1221 & C7101(3,1,4) \\
\hline 8 & C7101(3,2,2) & C7101(1,3,1) & C7101(1,1,1) \\
\hline 9 & C7101 $(1,1,2)$ & C7101(3,3,1) & - \\
\hline 10 & C7101(1,31) & C7101(1,2,4) & - \\
\hline 11 & C7101(2,3,1) & C7101(2,3,2) & - \\
\hline 12 & C7101(1,1,4) & C7101(3,1,4) & - \\
\hline 13 & RVF1 & C7101(2,2,1) & - \\
\hline 14 & SAREA-2 & C7101(2,2,2) & - \\
\hline 15 & C7101(2,2,4) & C7101(1,1,4) & - \\
\hline 16 & C7101(1,3,2) & DSF & - \\
\hline 17 & C7101(3,1,4) & C7101(1,1,1) & - \\
\hline 18 & C7101(2,3,4) & C7101(3,2,1) & - \\
\hline 19 & RVF2 & RVF2 & - \\
\hline 20 & BLC-2 & $7101(1,3,4)$ & - \\
\hline
\end{tabular}

The exchangeability property entails that the order of the information fed to the Bayesian updating process is irrelevant to the results (e.g., if the order of $B=100$ bootstrap replicates is shuffled to updating process leads, independently from the order of the evidences, to the same final ranking). Again for input variable 19, the ensemble of sensitivity measures calculated with the $\mathrm{B}=100$ and the same shuffled replicates, differ for less than $1 \%$, which proves that the proposed process possesses the exchangeability property.

This means that the Bayesian ensemble aggregation has to be preferred to Rsum and Rmv because it is robust to the variability of the experiments results to be used for sensitivity analysis as it allows cumulating knowledge (memory property) irrespectively of the occurrences sequence of the experiment results (exchangeability property). 


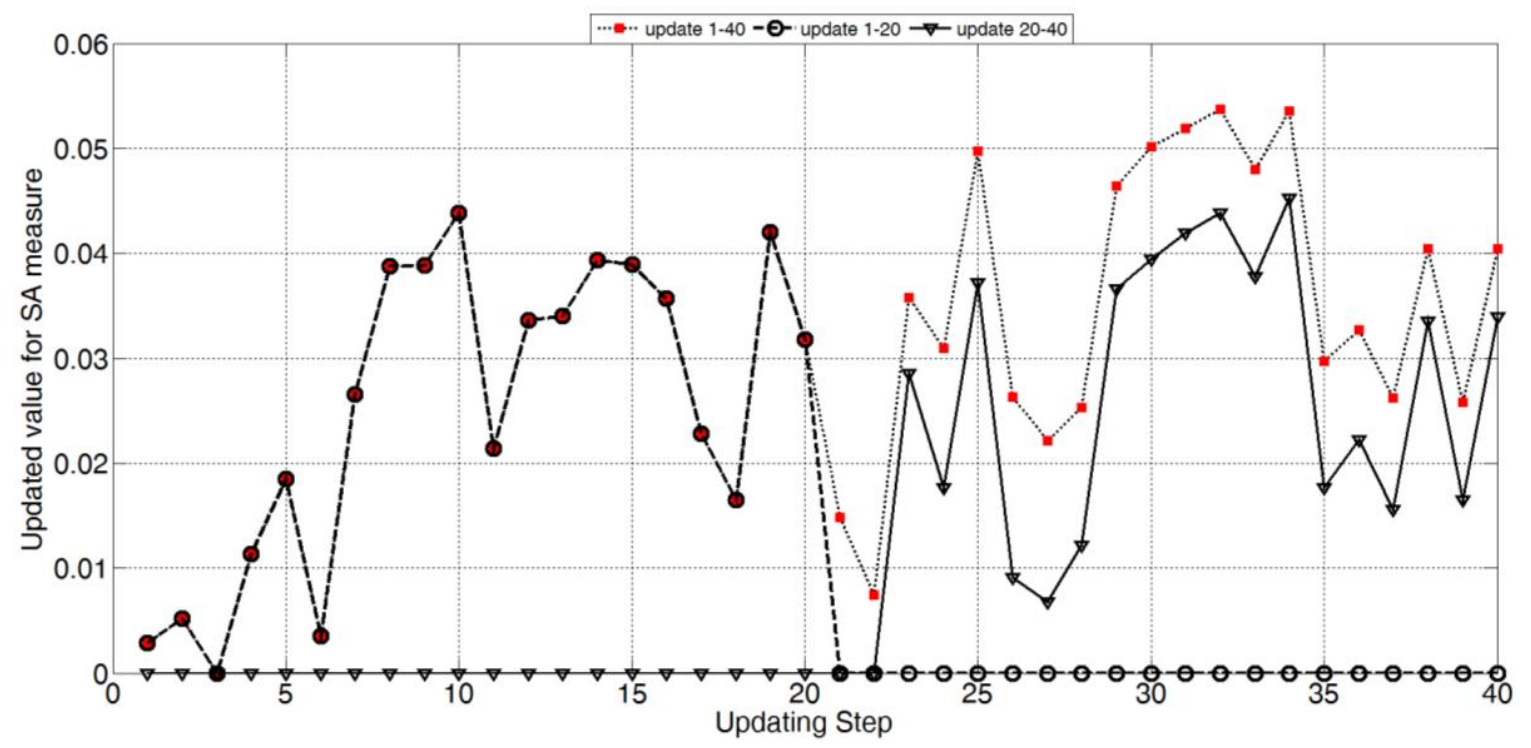

Fig. 7 Memory property of updating

\section{Conclusion}

In this paper, a novel framework is presented for performing an ensemble sensitivity analysis based on the Bayesian updating procedure. The framework is built based on performing an uncertainty analysis on the severe accident model for estimating a Gaussian FMM to retrieve the analytical pdf of the model output with as few simulations as possible. A bootstrap technique is adopted to replicate $B$ new model output datasets. An innovative ensemble strategy is designed to aggregate three sensitivity methods, namely input saliency, Hellinger distance and Kullback-Leibler divergence, that exploits a Bayesian updating procedure based on the Bradley-Terry algorithm for an incremental learning and iterative update of the input variables sensitivity measures. An application is shown with regards to a long-running MELCOR code simulating the fission product release during a LOCA in a scaled-down PWR. The results show the capability of the proposed framework in discerning between influent and negligible input variables, with properties of memory and exchangeability inherent in the Bayesian updating process.

\section{Nomenclature}

\begin{tabular}{|l|l|}
\hline COV & Coefficient Of Variation \\
\hline DSA & Deterministic Safety Assessment \\
\hline DOE & Design of Experiment \\
\hline ECCS & Emergency Core Cooling System \\
\hline EM & Expectation Maximization \\
\hline FMM & Finite Mixture Model \\
\hline FOM & Figure of Merit \\
\hline FP & Fission Products \\
\hline
\end{tabular}




\begin{tabular}{|l|l|}
\hline INL & Idaho National Laboratory \\
\hline LOCA & Loss of Coolant Accident \\
\hline LOFT & Loss of Flow Test \\
\hline LPIS & Low Pressure Injection System \\
\hline MV & Majority voting \\
\hline NPP & Nuclear Power Plant \\
\hline pdf & Probability density function \\
\hline PWR & Pressurized Water Reactor \\
\hline RF & Release fraction \\
\hline RN & Radio Nuclide \\
\hline SA & Sensitivity Analysis \\
\hline SNL & Sandia National Laboratories \\
\hline TH & Thermal-hydraulics \\
\hline
\end{tabular}

\section{References}

[Baraldi et al., 2011] P. Baraldi., E. Zio, G. Gola, D. Roverso, M. Hoffmann, "Two novel procedures for aggregating randomized model ensemble outcomes for robust signal reconstruction in nuclear power plants monitoring systems", Annals of Nuclear Energy 38, 212-220, 2011.

[Borgonovo, 2007] Borgonovo, E., “A new uncertainty importance measure”, Reliability Engineering and System Safety 92, 771-784, 2007.

[Cadini et al., 2007] F. Cadini, E. Zio, F. Di Maio, V. Kopustinkas, R. Urbonas, “A Neural-networkbased Variance Decomposition Sensitivity Analysis", International Journal of Nuclear Knowledge Management, Vol. 2, No. 3, pp. 299-312, 2007.

[Carlos et al., 2013] Carlos, S., Sánchez, A., Ginestar, D., Martorell, S., "Using finite mixture models in thermal hydraulics system code uncertainty analysis", Nuclear Engineering and Design 262, 306-318, 2013.

[Diaconis et al., 1982] P. Diaconis, Sandy L Zabell, “Updating subjective probability”, Journal of the American Statistical Association 77 (380), 822-830., 1982.

[Di Maio et al., 2012] F. Di Maio, J. Hu, W. Peter, M. Pecht, K. Tsui, E. Zio, "Ensemble approaches for clustering health status of oil sand pumps",Expert Systems with Applications 39, 4847-4859, 2012.

[Di Maio et al., 2014] F. Di Maio, G. Nicola, E. Zio, Y. Yu, "Ensemble-based sensitivity analysis of a Best Estimate Thermal Hydraulics model: Application to a Passive Containment Cooling System of an AP1000 Nuclear Power Plant", Annals of Nuclear Energy, vol. 73, p. 200-210, 2014.

[Di Maio et al., 2015] F. Di Maio, G. Nicola, E. Zio, Y.Yu, "Finite Mixture Models for sensitivity analysis of Thermal Hydraulic Codes for Passive Safety Systems safety Analysis", Nuclear Engineering and Design, 289, 144-154.

[Efron et al., 1993] B. Efron and T. R.J., "An Introduction to Bootstrap", New York: Chapman \& Hall/CRC, 1993.

[Figueiredo et al., 2002] M. Figueiredo, A.K. Jain, "Unsupervised learning of finite mixture models", IEEE Transactions on Pattern Analysis and Machine Intelligence 42 (3), 1-16, 2002. 
[Friedman et al., 1997] Friedman, N., Goldszmidt, M., "Sequential update of Bayesian network structure", UAI'97 Proceedings of the Thirteenth conference on Uncertainty in artificial intelligence, 165-174, 1997.

[Gibbs et al., 2002] A. L. Gibbs, Edward Su, "On choosing and bounding probability metrics", International Statistical Review 70 (3), 419-435, 2002.

[Helton, 1993] Helton, J.C., "Uncertainty and sensitivity analysis techniques for use in performance assessment for radioactive waste disposal", Reliability Engineering and System Safety 42, 327 367, 1993.

[Hoseyni et al., 2014] S. M. Hoseyni, M. Pourgol-Mohammad, A. Abbaspour Tehranifard , F. Yousefpour, "A Systematic Framework for Effective Uncertainty Assessment of Severe Accident Calculations; Hybrid Qualitative and Quantitative Methodology," Reliability Engineering and System Safety, vol. 125, pp. 22-35, 2014.

[Hoseyni et al., 2015] S. M. Hoseyni, M. Pourgol-Mohammad, "Model uncertainty in severe accident calculations: a structural methodology with application on LOFT LP-FP-2 experiment," Journal of Nuclear Technology, under review, 2015.

[Hunter, 2004] Hunter, D.R., "MM algorithms for generalized Bradley-Terry models", Annals of Statistics, 32 (1), pp. 384-406, 2004.

[Kmetyk, 1992] L. Kmetyk, "MELCOR 1.8.1 Assessment: LOFT Integral Experiment LP-FP-2", SAND92-1373, 1992.

[Kukkonen et al., 2007] S. Kukkonen, J. Lampinen, "Ranking-dominance and many-objective optimization", Evolutionary Computation, 3983-3990, 2007.

[IRSN-200783, 2007] IRSN, "Research and development with regard to severe accidents in pressurized water reactors; Summary and outlook", IRSN-200783, 2007.

[Law et al., 2004] Martin H.C Law, Mario A.T. Figueiredo, Anil K. Jain, "Simultaneous feature selection and clustering using mixture models", IEEE Transactions on Patterns Analysis and Machine Intelligence 26 (9), 1154-1166, 2004.

[Lewis et al., 2008] D. R. Lewis, B.J., F. Iglesias, Ducros and T. Kudoe, "Overview of experimental programs on core melt progression and fission product release behaviour", Jouranl of Nuclear Material, vol. 2008, pp. 126-143, 2008.

[Liu et al., 2010] Liu, Q., Homma, T., “A new importance measure for sensitivity analysis”, Journal of Nuclear Science and Technology, 47 (1), pp. 53-61, 2010.

[MacKay, 1995] MacKay, M.D.," "Evaluating Prediction Uncertainty", Los Alamos National Laborator Technical Report, NUREG-GE-6311, 1995.

[McLachlan et al., 2000] G. McLachlan and D. Peel, "Finite Mixture Models", New York: John Wiley \& Sons Inc., 2000.

[NEA, 2001] OECD/NEA, "In-Vessel Core Degradation Code Validation Matrix Update 19961999", NEA/CSNI/R(2000)21, 2001.

[Pengfei et al., 2014] W. Pengfei, Lu Zhenzhou and R. Wenbin S. Jingwen, "Regional Sensitivity Analysis Using Revised Mean and Variance Ratio Functions", Reliability Engineering \& System Safety, Vol. 121, pp. 121-135. Elsevier, 2014.

[Plischke et al., 2013] Plischke E., E. Borgonovo , C. L. Smith, "Global sensitivity measures from given data", European Journal of Operational Research, vol. 226, p. 536-50, 2013.

[Pourgol-Mohammad et al., 2007] Pourgol-Mohammad, M., Modarres, M., and Mosleh, A.,"Integrated Methodology for Thermal-Hydraulic Code Uncertainty Analysis with Application", Nuclear Technology, vol. 165, pp. 333-359, 2007. 
[Pourgol-Mohammad, 2009] Pourgol-Mohammad, M. "Thermal-hydraulics system codes uncertainty assessment: a review of the methodologies", Annals of Nuclear Energy 36, 17741786, 2009.

[Probst et al., 2006] J. Joucla, P. Probst, "Rank Statistics and Bootstrap: A More Precise Evaluation of the 95th Percentile in Nuclear Safety LB-LOCA Calculations", 14th Int. Conf. Nuclear Engineering (ICONE 14), Miami, Florida, 2006.

[Pudil et al., 1995] P. Pudil, J. Novovicova, J. Kittler, "Feature selection based on the approximation of class densities by finite mixtures of the special type", Pattern Recognition 28 (9), 1389-1398, 1995.

[Saltelli et al., 1999] Saltelli, A., Tarantola, S., Chan, K.P.-S., “A quantitative model-independent method for global sensitivity analysis of model output", Technometrics, 41 (1), pp. 39-56, 1999.

[Saltelli, 2002] Saltelli, A., "Sensitivity analysis for importance assessment", Risk Analysis, 22 (3), pp. 579-590, 2002.

[Secchi et al., 2008] P. Secchi, E. Zio and F. Di Maio, "Quantifying Uncertainties in the Estimation of Safety Parameters by Using Bootstrapped Artificial Neural Networks", Annals of Nuclear Energy, Vol. 35, pp. 2338-2350. Elsevier, 2008.

[Sobol et al., 1995] Saltelli, A., Sobol, I.M., "About the use of rank transformation in sensitivity analysis of model output", Reliability Engineering and System Safety, 50 (3), pp. 225-239, 1995.

[Vaithyanathan et al., 1999] S. Vaithyanathan, B. Dom, "Generalized model selection for unsupervised learning in high dimensions", Advances in Neural Information Processing Systems. MIT Press, Cambridge, Mass., pp. 970-976, 1999.

[Weng et al., 2011] R. C. Weng and C.-J. Lin, "A Bayesian Approximation Method for Online Ranking", Journal of Machine Learning Research, vol. 12, pp. 267-300, 2011.

[Zio et al., 2008] E. Zio and F. Di Maio, "Bootstrap and Order Statistics for Quantifying ThermalHydraulic Code Uncertainties in the Estimation of Safety Margins", Science and Technology of Nuclear Installations, Volume 2008 (2008), Article ID 340164, 9 pages, doi:10.1155/2008/340164. 